\title{
Multiple Correspondence Analysis on Rural Residents' Choices of Medical Institutions with Common Disease
}

\author{
Lili Zhu ${ }^{1,2,3, a}$, Changhai Tang ${ }^{1,2,3, b}$, Junwei Song ${ }^{1,2,3, ~ c}$, Zhiqiang Feng ${ }^{1,2,3}$, \\ ${ }^{\mathrm{d}}$, Qingzhu Wen ${ }^{1,2,3, \mathrm{e}}$, Zhongming Chen ${ }^{1,2,3, \mathrm{f}}$ and Wenqiang Yin ${ }^{2,3,4, \mathrm{~g} \text {, * }}$
}

\footnotetext{
${ }^{1}$ School of Public Health and Management, Weifang Medical University, Weifang 261053, China;

${ }^{2}$ Collaborative Innovation Center of Social Risks Governance in Health, Shanghai 200032, China.

3“'Health Shandong" Severe Social Risk Prevention and Management Synergy Innovation Center, Weifang 261053, China;
}

${ }^{4}$ Department of Public Course, Weifang Medical University, Weifang 261053, China.

allwfyxy@foxmail.com, ${ }^{b} 15056041071 @ 163 . c o m,{ }^{c}$ sjwsongjunwei@163.com, ${ }^{d}$ fengzhiqiang7@16 3.com, ${ }^{\mathrm{e}} 15269613219 @ 163 . c o m,{ }^{\mathrm{f}} \mathrm{Czm} 3306196 @ 163 . c o m,{ }^{9}$ yinwq1969@126.com.

* Corresponding author

Keywords: Choice of medical institutions, Multiple correspondence analysis

\begin{abstract}
Patients' choices of medical institutions reflect the flow direction of patients and requirements for different levels medical services. Reasonable distribution of medical services can improve health resource configuration structure and investing adjustment of health resource by redistributing medical services in different medical institutions. Method: This paper adopts multi-stage stratified sampling to pick out rural residents from six cities in Shandong province to make a research. Descriptive analysis has been applied to analyze the preferences with providers and their reasons of rural residents. Then multiple correspondence analysis (MCA) has been used to analyze different characteristics of medical institutions for rural residents. Results: The first choice of rural residents is rural clinic, which accounts for $57.2 \%$. The advantages of private clinics (440 points), rural clinics (1893 points) and township hospitals or community health service(CHS) institutions (444 points) are close distance and convenience, while the advantage of county hospitals (128 points) or other medical institutions (57 points) is high-level medical skills. It has a statistical meaning and choice differences based on different age, education background, income and employment status $(P<0.00)$. The characteristics of rural residents to choose different types of medical institutions are also different. Conclusion: It is necessary to increase invest in PHC institutions especially the construction of rural clinics. The service ability of PHC institutions should be improved. And to improve harmonious development, the construction of medical institutions both in county and township needs to be reasonably planned. Publication and guidance of reasonable medical concept to college students should also be intensified. Policy combination of perfecting essential medicine system, health care system and other medical service system should be improved to guide rural residents to form a reasonable and scientific choice of medical institutions.
\end{abstract}

\section{Introduction}

"Seeing a doctor is difficult and expensive" is a hot and focus issue in Chinese society. In 2009, The Opinions of the State Council of the Central Committee of the Communist of China on Deepening the Reform of Medical Health System was issued, and the reform of difficulty of seeing a doctor and high cost of seeing a doctor has been launched [1]. Since the carry out of new medical reform policy, it has had a different influence on the flow tendency of rural residents' patients no matter in the practice of essential medicine system or the improvement of medical insurance[2-3]. Parts of them improve the realization of reasonable choices of medical institutions, while others disturb the medical order. The Program of Health China 2030 was issued on October, 2016 by the 
Central Committee of the Communist Party of China and the State Council. It proposed that an integrated medical health service system should be built in a comprehensive way with an intact system, clear division of labor, complementary function, close cooperation and high efficiency, which can serve the people perfectly.

The most of current researches are inclined to choose the community as their research subjects [4-6], while the researches concerning rural are relatively few. However, the latest data show that rural population account for $45.23 \%$ of the whole population in China [7]. As a county with a large rural population, the improvement of rural residents' health condition can not only improve income, but also has a significance in increasing welfare level, which has a far-reaching influence on the construction of the whole country and economic development. This paper chooses rural residents as research subjects, analyses the common disease for hospital visits flow, acquaint with the choices of medical institutions of rural residents concerning common diseases and analyses its influencing factors, which can guide patients to form a reasonable choice of medical institutions and provide countermeasures and suggestions for perfecting medical health service system.

\section{Research Materials and Methods}

\subsection{Research Subject}

This study chooses rural residents of Shandong Province as research subjects. Multi-stage stratified sampling has been adopted to choose six cities including Jinan, Dongying, Linyi, Weifang, Binzhou and Dezhou based on different economic development. Among the six cities, three counties have been selected, and each county select 3 townships to choose rural residents. The number of questionnaires is 600 , collected 576, and including 555 valid questionnaires. The study researches 555 families of rural residents with a total number of 1612 .

\subsection{Research Content and Methods}

The questionnaires are made by our own research group. Self- administered questionnaires are used to in this study. The residents with old ages, low vision and low education background who cannot fill the questionnaires by themselves can ask the trained researchers to complete the form. The research content includes the common conditions of rural residents, health conditions and hospital visiting intention and so on.

\subsection{Statistical Data Analysis}

SPSS19.0 has been used to input data, and descriptive analysis has been adopted to analyze the basic situations of rural residents and reasons for different hospital visits intentions after an exact logic examination of computer. A Chi-square test is used to acquaint with choices of medical institutions of different rural residents. The study adopts MCA to analyze the variables with statistical differences in chi-square test and factors influencing rural residents' choices of medical institutions in a comprehensive way.

\section{Results}

\subsection{Sample Characteristics}

Female proportion is higher than male proportion, which account for $50.1 \%$. And the average ages are 38.32 \pm 19.07 , including 1347 residents who is above 15 years old. To have a reasonable analysis on choices of medical institutions of rural residents, the study only chooses the residents above 15 years old with an independent ability to choose. Among the residents above 15 years old, the marital status is mainly married which accounts for $81.9 \%$, while unmarried accounts for $15.2 \%$ and other status accounts for $2.9 \%$. As to education background, the education level is mainly junior middle school education which accounts for 39.1\%, while under elementary education including elementary education accounts for $23.7 \%$, senior high school or technical education accounts for $19.3 \%$ and junior college degree and above accounts for $17.9 \% .99 .7 \%$ residents have medical insurance, indicating high rate of medical insurance coverage. As to employment status, $65.4 \%$ rural residents are employed while $24.2 \%$ of them are still unemployed, $10.4 \%$ of rural residents are students. 


\subsection{The First Contact Care Intention of Rural Residents' Common Diseases}

The study shows that the choices of medical institutions of rural residents' common diseases are mainly rural clinics which accounts for $57.2 \%$ including 759 residents, while the intention of private clinics accounts for $13.6 \%$ including 181 residents, township hospitals or CHS institutions accounts for $15.8 \%$ including 210 residents, county hospitals accounts for $10.9 \%$ including 144 residents and intention of other medical institutions accounts for $2.5 \%$ including 33 residents. From the above data, we can see that the first choices of rural residents are mainly PHC institutions including private clinics, rural clinics, township hospitals/CHS institutions. However, almost 15\% rural residents would choose non-PHC institutions as their first choices.

\subsection{Reasons of Rural Residents' Choices of Medical Institutions}

Based on significance principle, the study divides the reasons of rural residents' choices of medical institutions into three items and ranks the three items. After calculating the scores of three items, the study gains the main reasons of rural residents' choices of medical institutions. The primary reason is close distance which is convenient for rural residents, while the reason of choosing non-PHC institutions is high-level medical skills. The details can be seen in table1.

Table 1 The first three reasons of rural residents' choices of medical institutions

\begin{tabular}{|c|c|c|c|c|c|c|c|c|c|}
\hline \multicolumn{2}{|c|}{ Private clinics } & \multicolumn{2}{|c|}{ Rural clinics } & \multicolumn{2}{|c|}{$\begin{array}{c}\text { Township } \\
\text { Hospitals/ } \\
\text { CHS institutions }\end{array}$} & \multicolumn{2}{|c|}{ County Hospitals } & \multicolumn{2}{|c|}{ Other institutions } \\
\hline Reasons & $\begin{array}{c}\text { Score } \\
\text { s }\end{array}$ & Reasons & $\begin{array}{c}\text { Score } \\
\text { s }\end{array}$ & Reasons & $\begin{array}{c}\text { Score } \\
\text { s }\end{array}$ & Reasons & Scores & Reasons & Scores \\
\hline $\begin{array}{c}\text { Close } \\
\text { distance/con } \\
\text { venience }\end{array}$ & 440 & $\begin{array}{c}\text { Close } \\
\text { distance/c } \\
\text { onvenienc } \\
\text { e }\end{array}$ & 1893 & $\begin{array}{c}\text { Close } \\
\text { distance/co } \\
\text { nvenience }\end{array}$ & 444 & $\begin{array}{l}\text { high-leve } \\
\text { l medical } \\
\text { skills }\end{array}$ & 265 & $\begin{array}{l}\text { High-leve } \\
\text { l medical } \\
\text { skills }\end{array}$ & 57 \\
\hline $\begin{array}{l}\text { Reasonable } \\
\text { charge }\end{array}$ & 193 & $\begin{array}{l}\text { Reasonabl } \\
\text { e charge }\end{array}$ & 755 & $\begin{array}{l}\text { High-level } \\
\text { medical } \\
\text { skills }\end{array}$ & 178 & $\begin{array}{c}\text { Close } \\
\text { distance/c } \\
\text { onvenien } \\
\text { ce }\end{array}$ & 128 & $\begin{array}{c}\text { Close } \\
\text { distance/c } \\
\text { onvenien } \\
\text { ce }\end{array}$ & 34 \\
\hline $\begin{array}{l}\text { High-level } \\
\text { medical } \\
\text { skills }\end{array}$ & 87 & $\begin{array}{l}\text { Good } \\
\text { attitude of } \\
\text { doctors }\end{array}$ & 365 & $\begin{array}{l}\text { Reasonabl } \\
\text { e charge }\end{array}$ & 169 & $\begin{array}{c}\text { Good } \\
\text { medical } \\
\text { equipmen } \\
\mathrm{t}\end{array}$ & 95 & $\begin{array}{c}\text { Good } \\
\text { medical } \\
\text { equipmen } \\
\mathrm{t}\end{array}$ & 23 \\
\hline
\end{tabular}

Scoring: The first one accounts for 3 scores, the second one accounts for 2 scores, and the third one for 1 score. The total number $=\sum$ scores*frequency

\subsection{Factors Influencing Rural Residents' Choices of Medical Institutions}

The study selects the variables affecting rural residents' choices of medical institutions based on the Chi-square test and professional knowledge. The study shows that there is a statistical meaning in different ages $\left(\chi^{2}=56.194, P<0.00\right)$, employment status $\left(\chi^{2}=29.544, P<0.00\right)$, education level $\left(\chi^{2}=108.396, P<0.00\right)$ and family income $\left(\chi^{2}=44.254, P<0.00\right)$. Therefore, all variables should be included in MCA. 


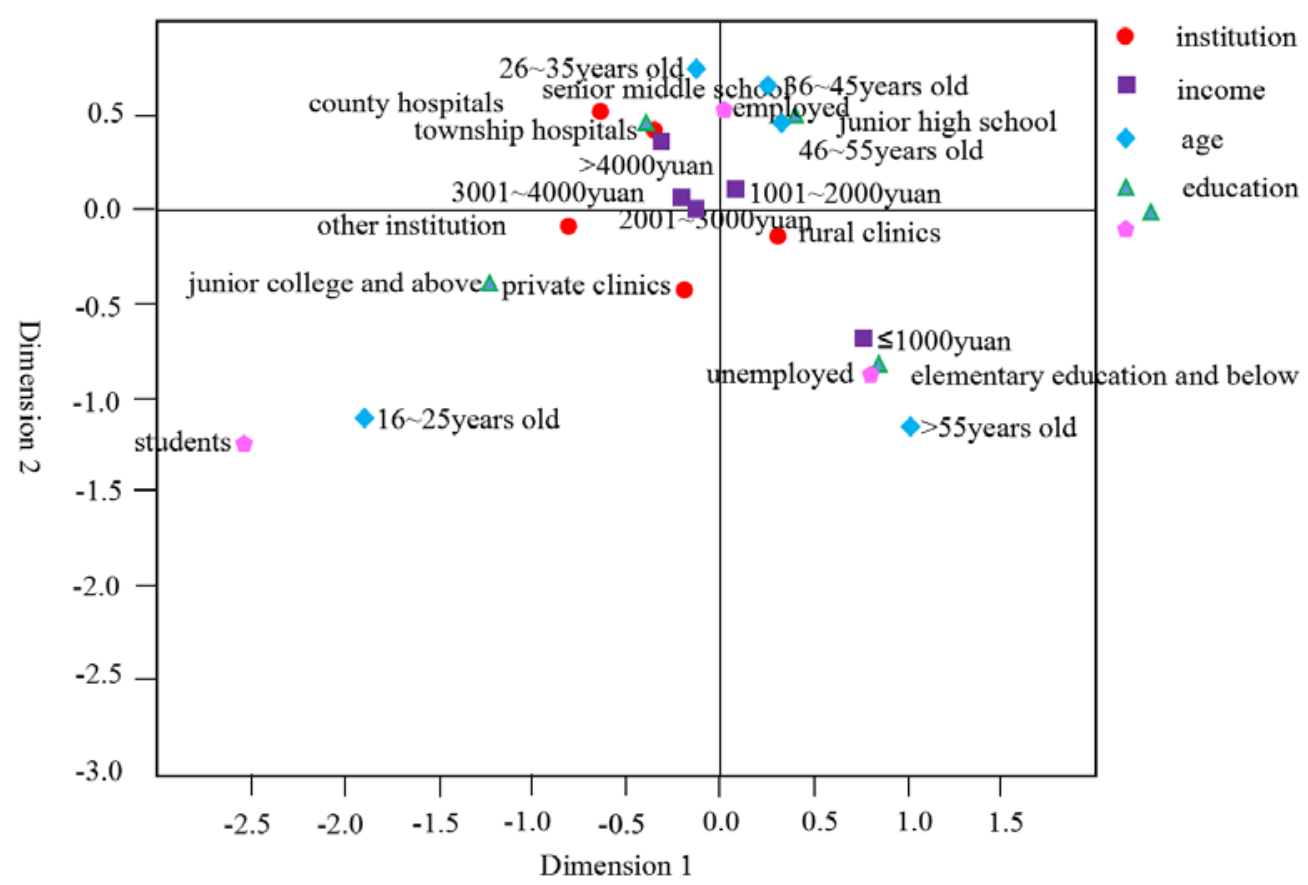

Fig. 1 Joint category plot of rural residents' choices for medical institutions with common disease

From the perspective of MCA analysis, a two-dimension MCA method is considered the most suitable. The first and second dimensions presented above are eigenvalue 2.380 and 1.812 respectively; inertia 0.476 and 0.362; and Cronbach's alpha 0.725 and 0.560. As it is shown in figure 1, the data of MCA indicates that the scatters concerning the choices of township hospitals/CHS institutions, county hospitals, family income above 4000yuan , senior high school/ technical secondary education and ages among 26 35years old have a close relation, while the scatters regarding family income below and including 1000yuan, ages above 55 years old, elementary education and unemployment status have a close relation, which have basically distributed from the original location(0.0) to line stretcher of rural clinic' scatters. The study connects the township hospital/CHS institutions and county hospitals and makes a ray from the original location. If the intersection angle between two line segments is small, it indicates that the two scattered points are homogeneous. The scatter concerning students with ages in 16 25 years old and junior college degree and above has a far distance from the original location (0.0) and there are no scattered points of institutions fall to the upper right quadrant. They have an inconspicuous characteristic in the requirement of selecting medical institutions.

\section{Discussion}

\subsection{Situation of First Contact Intention at PHC Institutions of Rural Residents}

As to common diseases, rural residents would mainly like to choose PHC institutions, and the proportion of selecting rural clinics is far higher than other medical institutions, which conforms to other researchers' results [8]. It can be clearly seen that the status of first options of PHC institutions of rural residents is better than that of urban residents, which result from the objective realities between rural and urban areas. In urban areas, there are high-level medical resources, convenient transportation, high service ability, high economic level, which enable urban residents to have better chances to select non-PHC institutions. However, in rural areas, with a population construction of stay-at-home children and old people, rural residents are inclined to choose PHC institution as their first options and pay much attention to the available medical services.

The main obstacles of unavailable medical service are the inconvenience to medical institutions. Among the reasons, close distance and convenience are the first options for rural residents, besides, low medical cost is also an important reason which has a close relation with low income of rural residents and high cost of big hospitals. When selecting county or other non-PHC institutions, 
residents would put high-level medical skills as their first option, which can be seen that rural residents have little confidence in medical level of PHC institutions. Rural residents treat PHC institutions as a kind of low cost and low quality service.

\subsection{Characteristics of Rural Residents to choose Rural Clinics}

As a basic unit of rural three-tier service network, rural clinics are the main institutions to guarantee the smoothly development of rural health work, and the health gatekeeper of rural residents as well. At present, the main patients of rural clinics are rural residents who have low income, low education background, unemployed and old aged. These patients who are stay-at-home old people with bad physical fitness and low ability to pay would prefer the nearby clinics to see a doctor. However, the situation of rural clinics is not satisfactory. There are a lack of doctors, more aging doctors and simple and crude medical equipment in rural clinics. The contradiction and conflict between stagnant medical service and increasingly requirements of medical services among rural residents have become fiercer and fiercer. The normalize construction of rural clinics improves the merge of many clinics and strengthen the administration to them, while it also brings inconveniences to the old patients and rural doctors when they go out to see a patient and lessens its main advantages of close distance.

\subsection{Characteristics of Rural Residents to choose County and Township hospitals}

When faced with common diseases, the group who choose township hospitals/CHS institutions or county hospitals is almost younger people and mainly from their own county or nearby counties. They are close to township hospitals, county hospitals and with a high income. The high income means the increase of ability to pay for a doctor, thus people would like to choose hospitals of high quality even they charge a lot [9]. However, with the improvement of people's living quality, the requirements for medical service have also be improved. Especially after the new medical reform, the compensation level of medical insurance has been improved. The average medical expense per visit has been decreased [10], and the medicine costs of patients has been decreased under the implementation of essential medicine systems [11]. However, the implementation of essential medicine system also restricts the development of medical business of PHC institution and the selection of medicine, in a long run, patients are doomed to flow into county or higher-level medical institutions [12], which affects the normal operation of all kinds of medical institutions and deviates from the original intention of new medical reform policy.

\subsection{Conflicts between the Development of County and Township Hospitals}

There is a competitive relationship between county and township hospitals. Acknowledged from interviews, there is an overlap of service group in township hospital and county hospitals. Because of high ability to gain resources and high ability of leaders, county hospitals are lower than PHC institutions, which leads to a blindly expansion of county hospitals and squeezes the existing room for township hospital and CHS institutions. And after the implementation of essential medicine system, because of lack of medicines, township hospitals have a lot of limitations in operating business which has a bad influence on their development. In the long run, it would lead to the flow of patients from PHC institutions to county hospitals, which increases the burden of rural residents, hospital visits disorder and is harmful to the construction of PHC system.

\subsection{Situations of Private Clinics and Other Medical Institutions}

Compared with other groups, the group who are students in 15 25 years old with junior college degree and above have wider options when seeing a doctor. It is probably because they are students who far away from their hometown with high education backgrounds, better physical condition and independent concepts to choose hospitals. Therefore, they may choose school clinics or buy drugs from nearby drugstores to cure themselves. Also, they may select some private clinics and other medical institutions. However, the group of college students pays little attention to hospital visits behaviors, and some colleges ignore the guidance of reasonable choices of seeing a doctor to students, which has a bad influence on the health behavior. 


\section{Conclusion}

\subsection{Service Ability of PHC Institutions Should Be Promptly Improved}

It has a high consciousness of first contact care intentions in PHC institutions among rural residents because of the objective reality of rural area. However, the medical service in rural area cannot meet the needs of rural residents and the service ability still needs to be improved, especially in medical skills and medical equipment. More invest should be put into PHC institutions by the government and other related departments. High quality resources should be shifted towards the primary level, and support should be increased. Besides, to provide better services to the rural residents.

\subsection{The Net Bottom of Rural Clinics Should Be Hold Up}

Rural clinic is the net bottom of PHC system, and it has great importance and cannot be ignored. The most important way is to increase the invest in rural clinics, improve the welfare of rural doctors. And government needs to take the responsibility. Role transformation should be strengthened. Doctors in rural clinics should be transformed into general practitioners. A reasonable construction of rural clinics should be taken. Combined with the rural reality, the construction should be planned in a convenient way to rural residents, avoiding the situation that administration is convenient while residents are inconvenient. Efforts should be taken to construct a better three-tier medical health service network as county hospitals are the chief, township hospitals are the pivot, and rural clinics are the basement. In this way, it can ensure the needs of rural residents in medical health service can be satisfied.

\subsection{Reasonable Planning of the Development of County and Township Hospitals}

Blindly expansion of county hospitals should be limited. A reasonable development plan should be established by scientific calculation about the past, present and future of medical service capacity. To release the essential medicine system is a good way to give a certain independence of the use of medicine to PHC institutions, which can satisfy the basic medicine use of PHC institutions and ensure the normal operation of medical service. It also can improve the scientific development of township hospitals and harmonious development of county and township hospitals, which can reduce malignant competition.

\subsection{Education in College Students' Health Behavior Should Be Enhanced}

Colleges should pay more attention to reasonable medical treatment of students, and strengthen publicity and education to the new students. Guidance on reasonable medical treatment should be given to students, which can avoid the waste of resources. Rural health work is an important part of China rural work. Whether it could be operated smoothly has a close relation with rural residents' healthy and agriculture development and social stability.

\section{Acknowledgment}

This work was financially supported by the National Natural Science Foundation of China (71173158 and 71373182). The authors would like to thank the National Natural Science Foundation of China for the funding of this research. Corresponding author of this paper is professor Wenqiang Yin.

\section{References}

[1] Lufa Zhang, Cheng Huang. Can the New Healthcare Reform Scheme Relieve "the Difficulty and High Cost of Getting Medical Service" Effectively in the Short Term, Population \& Economics ,2010, (5):43-51.

[2] Wenwen Liu, Rui Guo, Yu Liu, et al. Impact of "Zero-Profit Policy in Drug Sale" on Medication-taking Behavior of Community Residents in Beijing, Chinese General Practice, 2010, 13(8A):2433-2437. 
[3] Jiaojiao Zou, Zhaokang Yuan, Lianjun Zhang. Dynamic Changes of Rural Residents' Medical Service Utilization in Jiangxi Province, Chinese General Practice, 2014, 17(26):3153-3156.

[4] Yan Xin, Yingying Li, Xiaofei Xie, et al. Research on Influential Factors of Public's Health Care Choice in the Community Medical Institutions, The Chinese Health Service Management, 2016, (2):91-94.

[5] Na Li, Pei Zhou, Fang Wang, et al. Effect of Use Experience on Use Wishes of Community Health Service, Chinese Journal of Social Medicine, 2016, 33(5):467-470.

[6] Guodong Tian, Guangrong Wang, Shiye Jin, et al. Analysis of outpatients' willingness to accept the first treatment in the community of Zhabei District of Shanghai, Chinese Health Resources, 2014, 17(3):241-244.

[7] Compiled by National Bureau of Statistics of China, China Statistical Yearbook-2015, China Statistics Press.

[8] Lina Liu, Lingzhong XU, Xingzhou Wang, et al. Analysis of Rural Residents' Choice of Health Institutions and its Influencing Factors, Chinese Health Economics, 2006, 25(9): 26- 27.

[9] Mujun Wang. The Analysis of Hospital Choice and Factors for Medical Consultation: An Empirical Study in Five cities of China, Shandong University, 2008.

[10] Anning Ma, Changxiang Li, Yuqi Shen, et al. Study on the Changes of Hospitalization Costs for Patients in Township Hospitals before and after the Implementation of Essential Medicine System, Chinese Health Economics, 2014, 33(11):31-34.

[11] Fei Wang, Wenqiang Yin, Dongmei Huang, et al. Comparison of Drug Consumption in Township Hospitals before and after Implementation of National Essential Medicine System in Shandong Province, Chinese General Practice, 2013, 16(11A):3712-3714, 3718.

[12] Jinwei Hu, Wenqiang Yin, Hongwei Guo, et al. Study on the Choice and Utilization Changes of Medical Service in the New RCMs Residents under the Essential Medicine System, The Chinese Health Service Management, 2014, (9):694-696. 